\title{
Nuevos registros de especies del género Pristimantis (Anura: Craugastoridae) para el nororiente de Colombia
}

\author{
New records of species of genus \\ Pristimantis (Anura: Craugastoridae) from northeastern Colombia
}

\author{
Aldemar Alberto Acevedo Rincón*, Rosmery Franco Pallares*, \\ Karen Lizeth Silva Pérez*
}

Resumen

Colombia es uno de los países con la mayor riqueza de especies del género Pristimantis; sin embargo, existen vacíos en el conocimiento taxonómico, ecológico y en la distribución de muchas de ellas, en particular para la región nororiental de Colombia, zona que por su cercanía con Venezuela probablemente alberga especies aun no reportadas para Colombia. Objetivo: Reportar nuevas áreas de distribución de especies del género Pristimantis para Colombia. Metodología: Entre octubre de 2010 y abril de 2012 se realizaron muestreos de anfibios en cuatro localidades del departamento de Norte de Santander, Colombia. Los especímenes colectados fueron debidamente fijados y se les tomaron todas las medidas morfométricas para su posterior identificación mediante las descripciones originales y comparaciones con ejemplares depositados en colecciones biológicas. Resultados: Se amplía la distribución geográfica de cuatro especies: $P$. gryllus, $P$. melanoproctus, $P$. mondolfii y $P$. frater. Las tres primeras conocidas previamente sólo para los Andes de Venezuela y son registradas por primera vez para Colombia; $P$. frater, que se distribuye en la cordillera Oriental, por lo que nuestro registro representa el punto más al norte de su distribución conocida. Conclusión: Tres nuevos registros de Pristimatis para Colombia y una expansión de la distribución de una especie previamente conocida en el país, no obstante estas especies afrontan amenazas debido a la fragmentación de bosques e infección por Batrachochytrium dendrobatidis en las localidades reportadas en Norte de Santander.

Palabras clave: Anfibios, Conservación, Distribución, Norte de Santander, Quitridiomicosis.

\begin{abstract}
Colombia is one of the countries with the highest species richness of the genus Pristimantis, however, there are gaps in taxonomic, ecological knowledge and distribution of many of them, particularly in the northeastern region of Colombia, since by its proximity to Venezuela probably harbors species not yet reported for Colombia. Objective: To report new distribution records for species of Pristimantis in Colombia. Methodology: Between October 2010 and April 2012 amphibians sampling were conducted in four localities in the department of Norte de Santander. The collected specimens were properly preserved and all smorphometric measurements were made for identified by the original descriptions and comparisons with specimens deposited in biological collections. Results: We expand the geographic distribution of four species: P. gryllus, P. melanoproctus, P. mondolfii and P. frater. The first three previously known only to the Andes of Venezuela and are registered for the first time for Colombia; $P$. frater, which is distributed in the Eastern Cordillera of Colombia, so our record represents the northernmost point of its known distribution. Conclusion: Three new records of Pristimatis for Colombia and one range expansion of one species for the country, however these species face threats from forest fragmentation and infection with Batrachochytrium dendrobatidis in the localities reported in Norte de Santander.
\end{abstract}

Keywords: Amphibians, Conservation, Distribution, Chytridiomycosis, Norte de Santander.

Grupo de Investigación en Ecología y Biogeografía (GIEB), Universidad de Pamplona, Pamplona, Norte de Santander, Colombia. e-mail: bioaldemar@gmail.com Fecha recepción: Enero 4, 2014 Fecha aprobación: Mayo 7, 2014

Editor asociado: Jiménez AM 


\section{Introducción}

Las ranas del género Pristimantis se conocen por su desarrollo directo, el cual les permite ocupar una gran diversidad de hábitats, facilitando su amplia distribución en la región neotropical. Esta característica se refleja en las abundancias que las especies tienen a nivel local, en áreas con alta cobertura vegetal, mayor profundidad de hojarasca, alta humedad y temperaturas bajas (Crump 1974, Duellman 2005, Heinicke et al. 2007). La mayoría de las especies presenta actividad durante la noche, perchando en un estrato vertical, mientras que durante el día los individuos suelen estar refugiados dentro de la hojarasca y raíces de árboles (Miyamoto 1982, Blair y Doan 2009).

Se han registrado más de 400 especies de Pristimantis para el neotrópico (Frost 2013). Colombia, con más de 781 especies de anfibios (SIB 2013, Acosta-Galvis 2013), ostenta el mayor número de especies de Pristimantis, con 201; sin embargo, esta gran diversidad contrasta con el alto número de especies que están en alguna categoría de amenaza (78 especies; IUCN 2014, Acosta-Galvis 2013). A pesar de la gran diversidad de especies de Pristimantis reportadas para Colombia, muchas regiones poseen una limitada información sobre las amenazas, ecología, distribución e historia natural, registrando 39 especies de este género con datos deficientes (IUCN 2014). La falta de estudios que proporcionen información sobre historia natural, distribución, ecología y diversidad, han limitado la evaluación de los niveles de amenaza, lo cual repercute en un retraso del diseño de planes de manejo y estrategias de conservación en el país (Urbina-Cardona 2008).

En la actualidad, el estado de conocimiento sobre los Pristimantis del departamento de Norte de Santander se encuentra en fase preliminar. A la fecha se conocen seis especies de Pristimantis: P. anolirex (Lynch 1983), P. douglasi (Lynch 1996), P. lentiginosus (Rivero 1982), P. nicefori (Cochran y Goin 1970), $P$. prolixodiscus (Lynch 1978) y $P$. tubernasus (Rivero 1982). Esta carencia de registros se debe a múltiples factores, como la ausencia de grupos específicos de investigación y los problemas de orden público en varias zonas del departamento, que impiden el acceso a ellas.
Durante exploraciones realizadas en varias localidades de Norte de Santander entre el 2010 y 2012 registramos cuatro especies del género Pristimantis, de las cuales tres, $P$. gryllus (Barrio-Amorós et al. 2012), P. melanoproctus (Rivero 1982) y $P$. mondolfii (Rivero 1982) son nuevos registros para Colombia, especies que eran conocidas previamente solo para Venezuela; por su parte, P. frater (Werner 1899) solo se tenían reportes para los departamentos de Cundinamarca y Meta, ampliando así su distribución geográfica.

\section{Metodología}

Área de estudio. Los muestreos se realizaron en cuatro localidades del departamento de Norte de Santander (Figura 1): 1. Vereda El Salado (N $\left.7^{\circ} 18^{\prime} 20.07^{\prime \prime}, \mathrm{W} 72^{\circ} 22^{\prime} 21.73^{\prime \prime}\right)$, a una elevación de 2700 metros, a $5 \mathrm{~km}$ dirección este del municipio de Toledo; 2. Vereda Los Remansos, en la zona de amortiguación del Parque Nacional Natural (PNN) Tamá (N 7²1'23.69", W 72²8'18.84"), con una altitud entre los 2100-2400 m, ubicada a tres km dirección noroeste del municipio de Toledo. Estas dos localidades corresponden a bosques andinos y altoandinos y están representadas por un mosaico de potreros y parches de bosques surcados por diferentes quebradas, las cuales drenan el río Cobaría del municipio de Toledo. Las otras dos localidades se encuentran en el municipio de Pamplonita, al sur-oriente del departamento; 3. Quebrada La Tigra (N 7³1'45.47", W 72³8'3.70"), a 1100 m de altitud, ubicada en el km 49 vía Pamplona-Cúcuta, en la vereda Matajira; 4. Vereda el Manzano (N 7 32'.0 24.2", W $\left.72^{\circ} 38^{\prime} .020 .2^{\prime \prime}\right)$, también a una altitud de $1100 \mathrm{~m}$, ubicada en el km 42 vía Pamplona-Cúcuta. Las dos localidades presentan una temperatura media anual de $25^{\circ} \mathrm{C}$, atañe a las zonas de vida bosque húmedo premontano y bosque húmedo montano bajo (Holdridge 1971).

Muestreo. Entre octubre de 2010 y abril de 2012, se realizaron cuatro muestreos en cada de una de las localidades abarcando tres hábitats (bosque, quebrada y zonas abiertas) con recorridos diurnos de 09:00 a 12:00 y de 14:00 a 16:00 horas, y recorridos nocturnos entre 18:00 y 24:00 horas. Se aplicó el método de inspección visual con búsqueda libre (Crump y Scott 1994). 


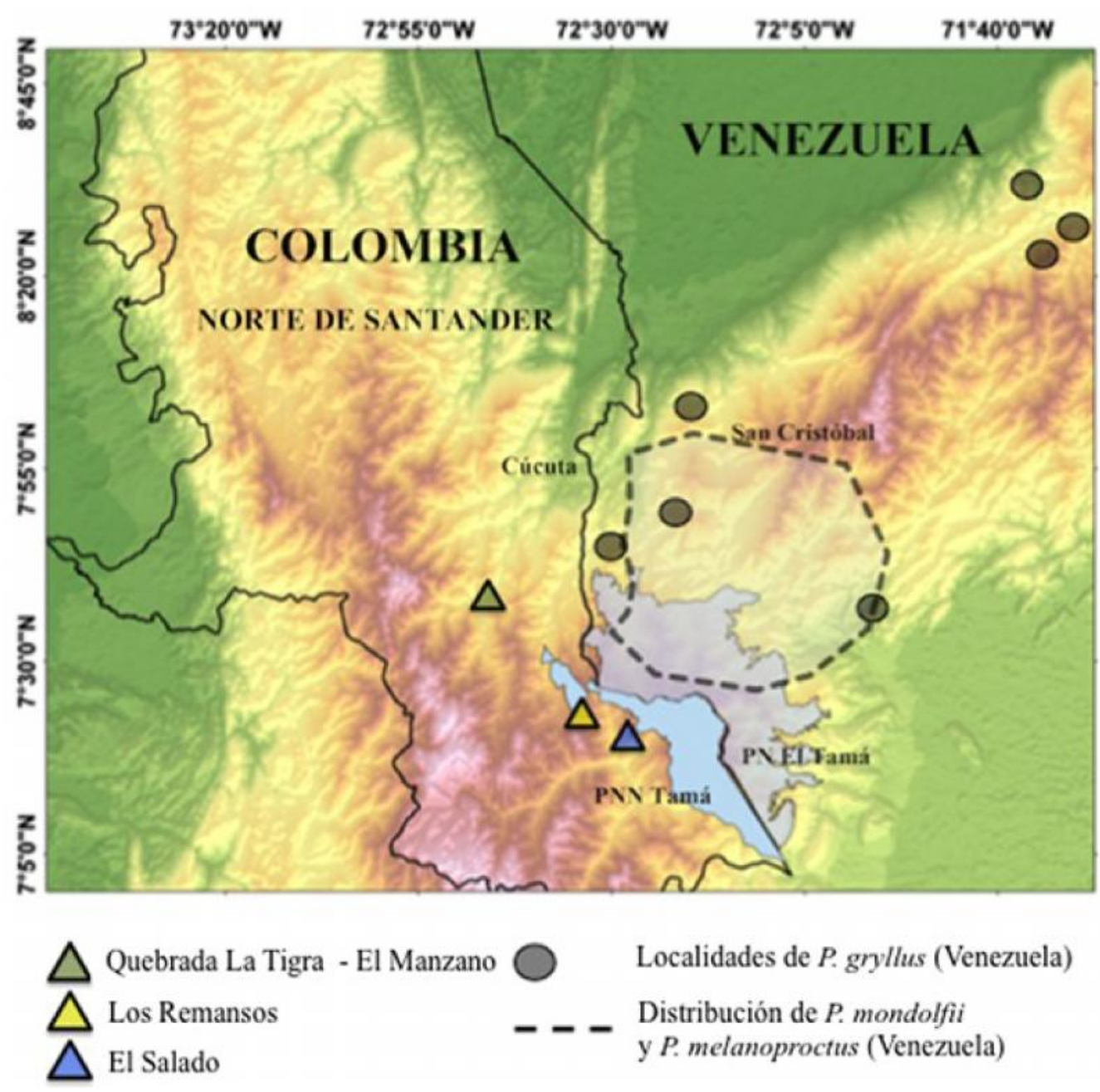

Figura 1. Mapa de Norte de Santander mostrando las localidades con registros para $P$. mondolfii, $P$. melanoproctus, P. frater (Los Remansos y El Salado) y P. gryllus (Quebrada La Tigra, Los Remansos y EI Salado).

Identificación de especímenes. Los ejemplares fueron capturados de forma manual y transportados individualmente en bolsas. Estos se preservaron en alcohol al 75\%. Luego se depositaron en la Colección Herpetológica del Museo de Ciencias Naturales de la Universidad de Pamplona (MCNUPH), P. gryllus (MCNUP-H 450-458), P. melanoproctus, (MCNUP-H 330-417), P. mondolfii (MCNUP-H 775-803) y P. frater (MCNUP-H 419421).

Se registraron medidas morfométricos de cada ejemplar con un calibrador con precisión $0,05 \mathrm{~mm}$. Las medidas tomadas fueron: longitud rostro-cloaca (LRC), ancho de la cabeza (AC), ancho de la boca (AB), longitud del fémur (LF), longitud de la tibia- fíbula (LTF), y longitud del antebrazo (LAB). Los especímenes fueron identificados a partir de las descripciones originales y esta identificación se corroboró revisando individuos depositados en la colección de referencia del Instituto de Ciencias Naturales (ICN) de la Universidad Nacional, Bogotá.

\section{Resultados}

\section{Nuevos registros para Colombia}

Pristimantis melanoproctus (Rivero 1982), Figura 2A. Con una distribución muy reducida, esta especie sólo se conocía en el estado Táchira, Venezuela, a unos $15 \mathrm{~km}$ del poblado las Delicias, a 1.800 metros de altitud. Se encuentra categorizada como 
datos deficientes (DD) (La Marca y García-Pérez 2004).

Se registró esta especie en las localidades de las veredas El Salado y Los Remansos, dentro del área de amortiguamiento del PNN Tamá, entre los 2100 y los $2700 \mathrm{~m}$ de altitud (Figura 1). Se registraron 46 individuos en bosques andinos y ribereños durante la noche, posados en arbustos entre uno y dos metros de altura al suelo.

Descripción y medidas morfológicas. Es una especie de tamaño mediano con un aparente dimorfismo sexual (machos LRC promedio de $12 \mathrm{~mm}$, hembras LRC promedio de $24,1 \mathrm{~mm}$; Tabla 1 ), hocico sub-elíptico, triangular y la punta ligeramente redondeada. El tímpano es moderadamente conspicuo y prominente. Presencia de coanas redondeadas y odontoides vomerinos moderadamente prominentes. Presenta el canto rostral de forma angular y ligeramente curva al llegar a la narina. El primer dedo más corto que el segundo con discos relativamente pequeños (Rivero 1982).

Pristimantis mondolfii (Rivero 1982), Figura 2B. Esta especie era conocida únicamente en los poblados de Matamula y Delicias, en el estado Táchira, Venezuela, a 1120 m de altitud. Rivero (1982) reportó, durante recorridos nocturnos, individuos de $P$. mondolfii sobre arbustos a no más de uno o dos metros del suelo, hacia el oeste de la depresión de San Cristóbal, Venezuela. La especie está categorizada como DD (La Marca y García-Pérez 2004) debido a la falta de información completa y detallada de la ecología, demografía, comportamiento e historia natural.
Registramos 67 individuos de esta especie por primera vez para Colombia, en las veredas El Salado y Los Remansos en inmediaciones del PNN Tamá, observando individuos a uno o dos metros de altura sobre la vegetación adyacentes a quebradas y bosques andinos, entre los 2100 y 2700 m de altitud.

Descripción y medidas morfológicas. Es una especie de tamaño mediano, con un aparente dimorfismo sexual (machos LRC promedio de 17,9 mm, hembras LRC promedio de 28,1 mm; Tabla 1), hocico subelíptico a veces ligeramente doblado hacia arriba y con la punta redondeada. El tímpano conspicuo y alargado dorso-ventralmente. Presenta odontoides vomerinos y coanas de forma ovaladas y oblicuas. Exhibe el canto rostral bien definido y angular. El primer dedo más corto que el segundo y muestran discos de tamaño moderado donde el disco del tercer dedo es tan grande como el tímpano (Rivero 1982).

Pristimantis gryllus (Barrio-Amorós et al. 2012), Figura 2C. Es una especie recién descrita, la cual se ha encontrado en siete localidades a lo largo de la parte suroccidental de la cordillera de Mérida y en los poblados de San Antonio, Pirineos y Matamula, en el estado Táchira, Venezuela. Se le considera como una especie común por su abundancia en los diferentes sitios. La especie recibe el nombre común de «ranita grillo andina», debido a que emite un canto distintivo que consistente en dos a cinco notas cortas parecidas a las de un grillo. Los machos se escuchan desde el atardecer hasta aproximadamente las 21:00 $\mathrm{h}$, en las zonas de bosque nublados a una altitud de 900-2020 m (Barrio-Amorós et al. 2012).

Tabla 1. Promedio de las medidas morfométricas en $\mathrm{mm}$ de las especies de Pristimantis reportadas en este estudio.

\begin{tabular}{|c|c|c|c|c|c|c|c|}
\hline Especies & LRC & AC & $A B$ & LAB & LF & LTF & Localidades \\
\hline P. melanoproctus (machos $=8$ ) & 12,0 & 5,2 & 4,8 & 2,7 & 3,6 & 5,9 & $\begin{array}{l}\text { El Salado, Los } \\
\text { Los Remansos }\end{array}$ \\
\hline P. melanoproctus (hembras $=8$ ) & 24,1 & 9,2 & 9,2 & 5,9 & 9,5 & 12,8 & \\
\hline P. mondolfii (machos $=12$ ) & 17,9 & 6,9 & 7,0 & 4,4 & 7,6 & 9,6 & $\begin{array}{l}\text { El Salado, } \\
\text { Los Remansos }\end{array}$ \\
\hline P. mondolfii (hembras $=5$ ) & 28,1 & 10,7 & 11,0 & 7,0 & 12,2 & 16,2 & \\
\hline P. gryllus (machos $=5$ ) & 11,8 & 4,0 & 4,0 & 3,0 & 5,5 & 6,1 & $\begin{array}{l}\text { El Salado, } \\
\text { Los Remansos y } \\
\text { quebrada La Tigra }\end{array}$ \\
\hline P. gryllus (hembras=6) & 20,4 & 7,3 & 6,1 & 4,4 & 9,7 & 10,6 & \\
\hline P. frater $($ machos $=4)$ & 15,9 & 5,1 & 5,5 & 3,6 & 6,12 & 7,1 & $\begin{array}{l}\text { El Salado } \\
\text { Los Remansos }\end{array}$ \\
\hline P. frater $($ hembras $=2$ ) & 21,2 & 8,1 & 7,1 & $4 ., 9$ & 10,1 & 11,2 & \\
\hline
\end{tabular}




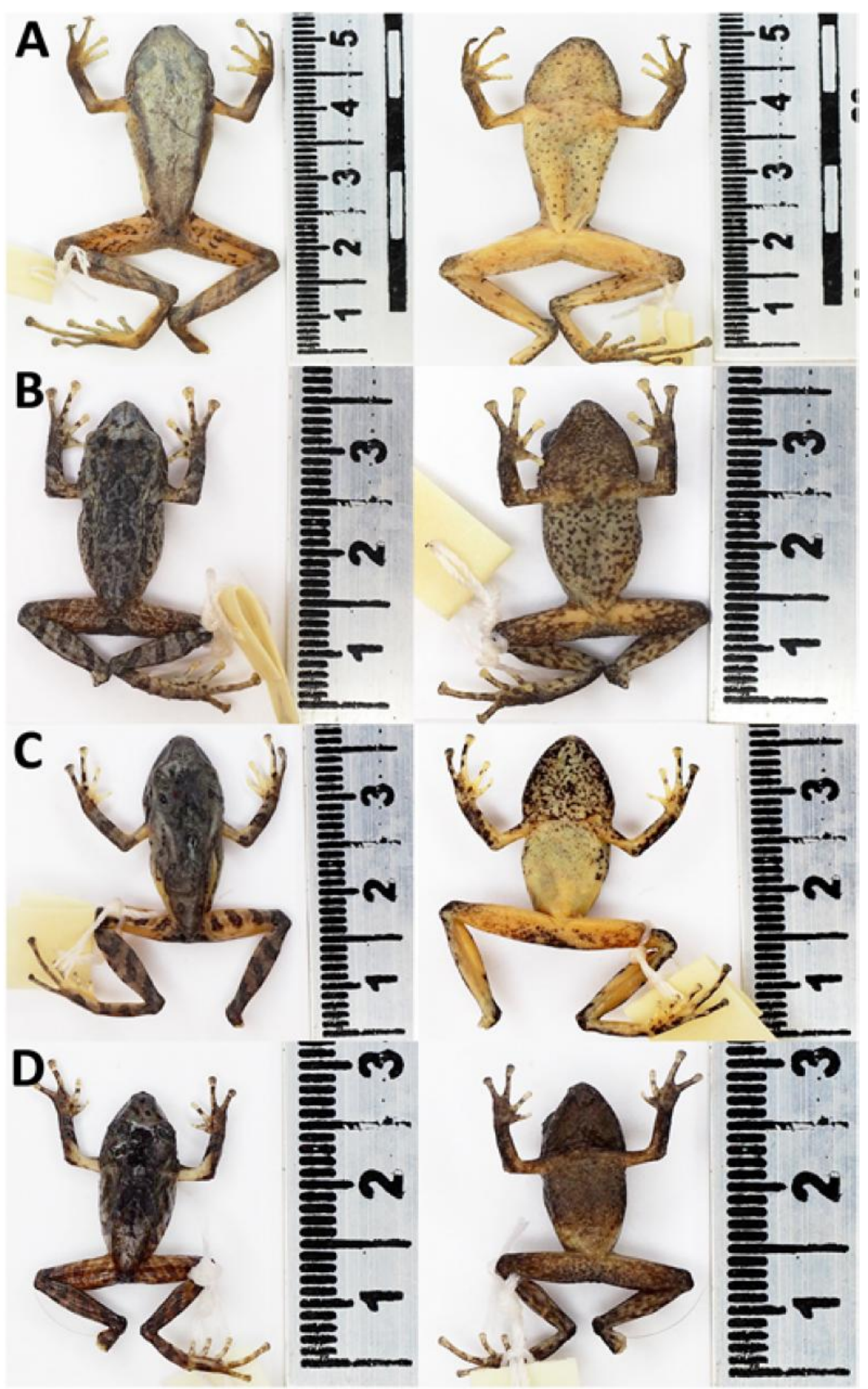

Figura 2. Vista dorsal y ventral de A. P. melanoproctus, B. P. mondolfii, C. P. gryllus y D. P. frater. 
El hallazgo de 22 individuos durante el presente trabajo de campo permite registrar por primera vez para Colombia esta especie. Se encontró a la rana grillo en tres localidades: dos en inmediaciones del PNN Tamá (veredas El Salado y Los Remansos), con bosques conservados e intervenidos, entre los 2100 a $2700 \mathrm{~m}$ de altitud; la tercera localidad corresponde a bosques ribereños de la quebrada La Tigra, vereda Matajira, municipio Pamplonita, km 49 vía Pamplona-Cúcuta (Figura 1) a 1200 m de altitud. Los individuos fueron observados a una altura máxima de dos metros sobre el suelo, perchados sobre la vegetación adyacente a quebradas, desde donde comenzaban a vocalizar entre las 18:00 y 19:00 horas disminuyendo su actividad cerca de las 23:00 horas.

Descripción y medidas morfológicas. Es una especie de tamaño pequeño, con un aparente dimorfismo sexual (machos LRC promedio de $11.8 \mathrm{~mm}$, hembras LRC promedio de 20,4 mm; Tabla 1), hocico redondeado en vista dorso-lateral. Presenta tímpano muy definido con anillo timpánico. Muestra coanas redondeadas. Exhibe canto rostral redondeado. Presenta hendiduras y saco vocales, sin almohadillas nupciales en los machos. Primer dedo es más corto que el segundo, con discos expandidos (BarrioAmorós et al. 2012).

Pristimantis frater (Werner 1899), Figura 2D. Esta especie se puede encontrar en la vertiente oriental de la cordillera Oriental de los departamentos de Cundinamarca y Meta y en la Serranía de la Macarena, en Colombia. Altitudinalmente, la especie se distribuye entre 1000 y $1600 \mathrm{~m}$ (Castro et al. 2010). El presente reporte constituye el primer registro de la especie para el departamento de Norte de Santander, ampliando su distribución al flanco este de la cordillera Oriental. Está categorizada como una especie vulnerable (VU) (Castro et al. 2010). En la zona evaluada se registraron ocho individuos en el remanente ribereño de las localidades El Salado y Los Remansos, por lo cual $P$. frater se considera como una especie rara.

Descripción y medidas morfológicas. Adultos de tamaño pequeño, con un aparente dimorfismo sexual (machos LRC promedio de 15,9 mm, hembras LRC promedio de 21,2 mm; Tabla 1). Zona baja del dorso recubierta con granulaciones, con grandes tubérculos aislados en el párpado superior, entre los ojos y en la región escapular, cabeza tan ancha como el cuerpo, sin crestas craneales, hocico corto subacuminado en vista dorsal y redondeado en vista lateral; el canto rostral poco definido y obtuso, tímpano prominente con 1/4 a 2/5 la longitud del ojo, piel del dorso finamente áspera, con verrugas dispersas, sin pliegues dorso laterales. Cojinetes truncados y pequeños; almohadillas grandes sobre los dedos exteriores, machos con almohadilla blanca nupcial, no espinosa. Primer dedo más corto que el segundo, pequeños tubérculos en el talón, dos tubérculos metatarsales (Pyburn y Lynch 1981).

\section{Discusión}

El departamento de Norte de Santander es una de las zonas con más vacíos de información sobre la fauna anfibia, donde sólo se conoce 5.3\% (40 spp) de los anfibios de Colombia (Acosta-Galvis 2000, Armesto et al. 2009, Acevedo et al. 2011, Acevedo et al. 2013b). No obstante, esta situación incrementa las posibilidades de encontrar nuevas especies de anfibios para la ciencia y ampliar el conocimiento sobre la distribución geográfica de otras especies.

Los Andes de Colombia y de Venezuela constituyen una continuidad de condiciones climáticas y ecológicas, por lo cual hay una alta probabilidad de que muchas de las especies de fauna y flora reportadas en el nororiente colombiano presenten distribuciones continuas entre la región andina y, por ende, se refleje en una similitud de composición de especies para los dos países. No obstante, el conocimiento sobre la composición y distribución de las especies de anfibios para estas regiones es limitado.

Pristimantis mondolfii y P. melanoproctus están reportadas por la IUCN como especies en DD, mientras que $P$. gryllus aun no ha sido evaluada, por lo que es prioritario realizar estudios ecológicos que permitan conocer el estado actual de sus poblacionales y su grado de vulnerabilidad a las diferentes amenazas.

Comparación entre P. melanoproctus y $P$. mondolfii. A partir de la descripción original de Rivero (1982), se logró corroborar las diferencias morfológicas entre $P$. melanoproctus y $P$. mondolfii, las cuales comparten aspectos similares como la forma del hocico y en la presencia de la membrana interdigital, pero sólo P. melanoproctus tiene repliegues dorsolaterales y una mancha negra en la abertura cloacal. Los discos de los P. melanoproctus se nota una leve 
ondulación mientras que $P$. mondolfii tiene los discos más truncos. La forma del tímpano de P. melanoproctus es ovalado, hundido, de márgenes ligeramente sobresalientes y de márgenes cubiertos en la región posterodorsal en $P$. mondolfii. Los procesos odontoides vomerianos son oblicuos, están bastante distantes de las coanas y no se unen en el medio en $P$. mondolfii mientras que los de $P$. melanoproctus son triangulares, comienzan al nivel del borde posterior de las coanas y no se unen en el medio (Rivero 1982).

Estado de conservación. Las cuatro especies de Pristimantis reportadas para el departamento de Norte de Santander, enfrentan amenazas, como el deterioro de hábitat causado por actividades antropogénicas (agricultura, ganadería y minería), sumado a esto, las especies de las tres localidades (El Salado, Los Remansos y Quebrada la Tigra) se encuentran infectadas por el hongo Batrachochytrium dendrobatidis (Acevedo et al. 2013a, 2013b, Franco 2013). Este hongo patógeno produce la quitridiomicosis, considerada como una enfermedad emergente y una de las principales responsables de la extinción de muchas especies de anfibios a nivel mundial (Berger et al. 1998, Daszak et al. 1999, Lips 1999, Retallick et al. 2004, La Marca et al. 2005).

Para comprender y determinar el patrón de distribución del patógeno en Norte de Santander, es necesario realizar estudios de incidencia y prevalencia en otras localidades del departamento, que permitan determinar el estado poblacional de los anfibios de las localidades reportadas como positivas. Por ello, es necesario implementar programas de monitoreo frecuentes, que contribuyan al conocimiento sobre la diversidad y estrategias de conservación adecuadas que mitiguen la pérdida de la biodiversidad de anfibios de la cordillera Oriental colombiana (UrbinaCardona 2008).

\section{Agradecimientos}

Agradecemos a Conservation Leadership Programme, Save Our Species, Idea Wild y a la Universidad de Pamplona por el financiamiento de este trabajo. A Orlando Armesto y a las familias residentes en las localidades de estudio por su apoyo en campo. Agradecemos de manera especial al profesor John D. Lynch de la Universidad Nacional de Colombia por la verificación e identificación taxonómica de las especies de Pristimantis reportadas en este estudio.

\section{Literatura citada}

Acosta-Galvis AR. 2000. Ranas, salamandras y caecilias (Tretrapoda: Amphibia) de Colombia. Biota Colomb. 1 (3): 289-319.

Acosta-Galvis AR. 2013. Lista de los anfibios de Colombia. V.02.2013.0. (En línea) 2014 (Acceso 20 de marzo). URL disponible en: www.batrachia.com

Armesto O, Esteban JB, Torrado R. 2009. Fauna de anfibios del municipio de Cúcuta, Norte de Santander. Herpetotropicos. 5 (1): 57-63.

Acevedo A, Silva K, Franco R. 2011. Distribución, historia natural y conservación de una rana marsupial poco conocida, Gastrotheca helenae (Anura: Hemipractidae), en el Parque Nacional Natural Tamá, Colombia. Bol Cient Mus Hist Nat U Caldas. 15 (1): 68-74.

Acevedo A, Franco R, Silva K. 2013a. Amphibians of the Tama National Park: Hidden biodiversity and New Salamander species from Colombia. FrogLog. 21 (106): 53-5.

Acevedo A, Wake D, Márquez R, Silva K, Franco R, Amezquita A. 2013b. Two new species of salamanders, genus Bolitoglossa (Amphibia: Plethodontidae), from the eastern Colombian Andes. Zootaxa. 3609 (1): 69-84.

Blair C, Doan TM. 2009. Patterns of community structure and microhabitat usage in Peruvian Pristimantis (Anura: Strabomantidae). Copeia. 2009 (2): 303-12.

Barrio-Amorós C, Guayasamin JM, Hedges B. 2012. A new minute Andean Pristimantis (Anura: Strabomantidae) from Venezuela. Phyllomedusa. 11 (2): 83-93.

Berger L, Speare R, Daszak P, Green DA, Cunningham AA, Goggin CL, et al. 1998. Chytridiomycosis causes amphibian mortality associated with population declines in the rain forests of Australia and Central. Am Proc Natl Acad Sci USA. 95: 9031-6.

Castro F, Herrera MI, Rueda JV. 2010. Pristimantis frater. IUCN Red List of Threatened Species (En línea) 2014 (Acceso abril 17). URL disponible: www.iucnredlist.org

Cochran DM, Goin CJ. 1970. Frogs of Colombia. Chicago: National Museum Bulletin Smithsonian Institution Press; $655 \mathrm{p}$.

Crump ML, Scott NY. 1994. Visual encounter survey. In: Measuring and monitoring biological diversity: Standard methods for amphibians. Heyer W, Donnelley MA, Mcdiarmid RA, Hayek LC, Foster MC. (eds.) Washington, DC: Smithsonian Institution; 364 pp.

Crump ML. 1974. Reproductive strategies in a tropical anuran community. Kansas: Miscellaneous Publication, University of Kansas Museum of Natural History; 68 $\mathrm{pp}$.

Daszak P, Berger L, Cunningham AC, Hyatt AD, Green DE, Speare R. 1999. Emerging infectious diseases and amphibian population declines. Emerg Infect Dis. 5: 73548.

Duellman WE. 2005. Cusco amazónico: The lives of amphibians 
and reptiles in an Amazonian rainforest. New York: Cornell University Press; 488 pp.

Franco R. 2013. Diversidad y estado de conservación de los anfibios andinos del Parque Nacional Natural Tamá, Colombia. (Trabajo de grado). Pamplona: Universidad de Pamplona. 95 pp.

Frost DR. 2013. Amphibian species of the World: an online reference. Version 5.6 (En línea) 2014 (Acceso 12 de marzo). URL disponible en: http://research.amnh.org/ herpetology/amphibia/index.html

Heinicke MP, Duellman WE, Hedges SB. 2007. Major Caribbean and Central American frog faunas originated by ancient oceanic dispersal. Proc Nat Acad Sci USA. 104 (24): 10092 7.

Holdridge LR. 1971. Forest enviroments in tropical life zones: A pilot study. New York: Pergamon Press; $747 \mathrm{pp}$.

IUCN. 2014. IUCN Red List of Threatened Species. V.2014.2. (En línea) 2014 (Acceso 18 de marzo). URL disponible en: www.iucnredlist.org

La Marca E, García-Pérez JE. 2004. Pristimantis melanoproctus. IUCN Red List of Threatened Species (En línea) 2014 (Acceso 17 de abril). URL disponible en: www.iucnredlist.org

La Marca E, Lips KR, Lotters S, Puschendorf R, Ibañez R, Rueda-almonacid JV, et al. 2005. Catastrophic population declines and extinction in Neotropical harlequin frogs (Bufonidae: Atelopus). Biotropica. 37: 90-201.

Lips KR. 1999. Mass mortality and population declines of anurans at an upland site in western Panama. Conserv Biol. 13: 117-25.
Lynch JD. 1978. A new Eleutherodactylinae frog from the Andes of Northern Colombia. Copeia. 1978: 17-21.

Lynch JD. 1983. A new leptodactylid frog from the cordillera Oriental of Colombia. Nebraska: School of Life Sciencies, University of Nebraska; 57 pp.

Lynch JD. 1996. New frog (Eleutherodactylus: Leptodactylidae) from the Andes of eastern Colombia, part of a remarkable pattern of distribution. Copeia. 1996: 103-8.

Miyamoto MM. 1982. Vertical habitat use by Eleutherodactylus frogs (Leptodactylidae) at two Costa Rican localities. Biotropica. 14 (2): 141-4.

Pyburn WF, Lynch JD. 1981. Two little-known species of Eleutherodactylus (Amphibia: Leptodactylidae) from the Sierra de la Macarena, Colombia. Proc Biol Soc Wash. 94: 404-12.

Retallick R, McCallum H, Speare R. 2004. Endemic infection of the amphibian chytrid fungus in a frog community postdecline. PLOS Biology. 2 (11): 1-7.

Rivero JA. 1982. Los Eleutherodactylus (Amphibia, Leptodactylidae) de los Andes venezolanos. II. Especies subparameras. Mem Soc Cien Nat La Salle. 42 (118): 57 132.

SIB. 2013. Sistema de Información sobre Biodiversidad de Colombia. (En línea) 2013 (Acceso 10 de abril) URL disponible en: http://www.sibcolombia.net/web/sib/cifras

Urbina-Cardona JN. 2008. Conservación de la herpetofauna neotropical: líneas de investigación y desafíos. Trop Conserv Sci. 1 (4): 359-75.

Werner F. 1899. Ueber Reptilien und Batrachier aus Columbien und Trinidad. Verh K K Zool-Bot Ges. Wien 49: 470-84. 